\title{
Intrinsic optical anisotropy of [001]-grown short-period InAs/GaSb superlattices
}

\author{
L. L. Li, ${ }^{1,2}$ W. Xu, ${ }^{2,3, *}$ and F. M. Peeters ${ }^{1, \dagger}$ \\ ${ }^{1}$ Department of Physics, University of Antwerp, Groenenborgerlaan 171, B-2020 Antwerpen, Belgium \\ ${ }^{2}$ Key Laboratory of Materials Physics, Institute of Solid State Physics, Chinese Academy of Sciences, Hefei 230031, China \\ ${ }^{3}$ Department of Physics, Yunnan University, Kunming 650091, China
}

(Received 5 October 2010; published 13 December 2010)

\begin{abstract}
We theoretically investigate the intrinsic optical anisotropy or polarization induced by the microscopic interface asymmetry (MIA) in no-common-atom (NCA) InAs/GaSb superlattices (SLs) grown along the [001] direction. The eight-band K·P model is used to calculate the electronic band structures and incorporates the MIA effect. A Boltzmann equation approach is employed to calculate the optical properties. We found that in NCA InAs/GaSb SLs, the MIA effect causes a large in-plane optical anisotropy for linearly polarized light and the largest anisotropy occurs for light polarized along the [110] and [1 $\overline{1} 0]$ directions. The relative difference between the optical-absorption coefficient for [110]-polarized light and that for [1 $\overline{1} 0]$-polarized light is found to be larger than $50 \%$. The dependence of the in-plane optical anisotropy on temperature, photoexcited carrier density, and layer width is examined in detail. This study is important for optical devices which require the polarization control and selectivity.
\end{abstract}

DOI: 10.1103/PhysRevB.82.235422

PACS number(s): 78.67.Pt, 73.21.Cd, 78.40.Fy

\section{INTRODUCTION}

Many of the optoelectronic devices used in modern optical communication systems require a polarizationindependent operation, whereas for laser diodes polarizationdependent mechanisms have been explored that reduce the threshold current. In recent years optical polarization phenomena in vertical-cavity surface-emitting lasers (VCSLs) have been intensively studied both experimentally and theoretically. ${ }^{1-3}$ In addition to VCSLs, some particular III-V semiconductor superlattices (SLs) have also attracted much interest in light of such phenomena. ${ }^{4-6}$

In the $C 1 A 1 / C 2 A 2$ semiconductor SLs where $C 1 / C 2$ and $A 1 / A 2$ denote, respectively, the III-V cation and anion species and if the host materials $C 1 A 1$ and $C 2 A 2$ have no common atoms (NCAs), i.e., $C 1 \neq C 2$ and $A 1 \neq A 2$, we will term such SLs as NCA SLs. In the other case when the host materials share common atoms, i.e., $C 1=C 2$ or $A 1=A 2$, we will call them CA SLs. Hence, in NCA SLs, there exists a remarkable peculiarity that chemical bonds formed at the interfaces $(C 1-A 2$ and $C 2-A 1)$ are different from those formed in the host materials ( $C 1-A 1$ and $C 2-A 2)$. Typical NCA systems are GaAs/InP and InAs/GaSb SLs while GaAs/AlAs and GaAs/InAs SLs are typical CA SL systems. The difference between the CA and NCA SLs comes from the inequivalent chemical bonds formed in the host materials and at their interfaces. Owing to this difference, the NCA SL system such as GaAs/InP has been intensively studied in the past decade to explore interface related electronic and optical properties. ${ }^{4-7}$ The most different features exhibited by CA and NCA SLs are seen in their optical properties. It has been experimentally verified that in the GaAs/InP NCA system a giant in-plane anisotropy (or polarization) in the optical absorption of light polarized along the [110] and [1 $\overline{1} 0]$ directions ${ }^{5,6}$ occurs while CA SLs do not show any in-plane anisotropy of their optical absorption.

It is not hard to understand the in-plane absorption anisotropy of NCA SLs grown along the [001] direction. In the
CA SLs such as GaAs/AlAs, successive interfaces have the following spatial arrangement of chemical bonds: the first interface has Ga-As and Al-As bonds lying in the (110) plane while the second has Al-As and Ga-As bonds lying in the (110) plane. As a result, the positive anisotropy induced by different Ga-As and Al-As bonds lying in the (110) plane is exactly compensated by the negative anisotropy resulting from different Al-As and Ga-As bonds lying in the (1 $\overline{1} 0)$ plane and thus no in-plane anisotropy occurs. However, this compensation does not exist in the NCA SLs such as GaAs/ InP: the first interface has Ga-As and In-As bonds lying in the (110) plane while the second has In-P and Ga-P bonds lying in the (110) plane. As a result, two in-plane directions $[110]$ and $[1 \overline{1} 0]$ are inequivalent and thus exhibits a strong in-plane anisotropy. When linearly polarized light propagates along the [001] SL growth direction, it will feel different chemical bonds for the [110] and [1 $\overline{1} 0]$ polarization directions and consequently, in-plane absorption anisotropy is induced by [110] and [1 $\overline{1} 0]$ polarized light. From the group symmetry point of view, CA SLs correspond to the $D_{2 d}$ point-group symmetry while NCA SLs correspond to the $C_{2 v}$ point-group symmetry. The $C_{2 v}$ interface symmetry in NCA SLs is lower than the $D_{2 d}$ interface symmetry in the CA SLs. Thus, the optical anisotropy induced by the microscopic interface asymmetry (MIA) effect is an intrinsic property of the NCA SL system and detailed studies of the in-plane optical polarization or anisotropy are necessary.

InAs/GaSb NCA SLs have attracted considerable interest over the past two decades due to their unique type-II band alignment and their application as detectors and lasers with tunable infrared wavelengths. ${ }^{8,9}$ Most of those studies focused on the electronic ${ }^{10-12}$ and spintronic ${ }^{13,14}$ properties of InAs/GaSb NCA SLs but only few works dealt with the optical anisotropy that intrinsically exists in such SL systems. Although the MIA influence on the in-plane optical polarization (the polarization rate up to $40 \%$ ) is well described for $\mathrm{GaAs} / \mathrm{InP}$ NCA structures, ${ }^{5,6}$ the situation in the InAs/GaSb 
NCA SL system is still unclear and is expected to be complicated because of a strong conduction-valence-band mixing which is absent in the GaAs/InP SL system. In addition, the InAs/GaSb SL system is the best type-II candidate: electrons and holes are, respectively, confined in the InAs and $\mathrm{GaSb}$ layers. Due to this fact, it is expected that the optical properties in such type-II NCA SLs should be extremely sensitive to the MIA effect because they depend completely on the overlap between the electron and the hole wave functions at the interface. Thus, one may anticipate that new phenomena related to the optical anisotropy could take place in the InAs/ GaSb NCA SLs.

The InAs/GaSb NCA SL system has been modeled using a variety of theoretical approaches such as the empirical tight-binding method, ${ }^{15,16}$ the empirical pseudopotential method, ${ }^{10,11}$ and the standard envelope function approximation (EFA) method. ${ }^{17,18}$ Among these approaches, the EFA method is most widely used because of its greater physical appeal, easier code implementation, and rather good numerical accuracy. However, it has been well known that the standard EFA method fails to distinguish the CA SL system from the NCA SL system when considering their optical properties: both the CA and NCA SLs do not show any in-plane optical polarization in the frame of standard EFA. Therefore, a more accurate approach is needed in which the MIA effect is taken into account in NCA SLs. In the literatures, there were many proposals for taking this effect into account in the EFA theory. Krebs and Voisin proposed a phenomenological model $^{4}$ called the $H_{\mathrm{BF}}$ model that allows to distinguish the interface chemical bonds that are stacked in backward (B) and forward $(\mathrm{F})$ directions. General boundary conditions were proposed ${ }^{19}$ for the multiband effective-mass theory in which the hole envelop function boundary conditions are generalized and mix the heavy-hole and light-hole spin components. Szmulowicz et al. ${ }^{12}$ developed a modified eightband EFA formalism that incorporate the MIA effect. It was recently shown that a nonsymmetrized eight-band $\mathbf{K} \cdot \mathbf{P}$ Hamiltonian $^{20}$ can also model the MIA effect within the Burt-Foreman theory. ${ }^{21}$ More recently, the MIA effect in InAs/GaSb SLs has been modeled successfully within the framework of the eight-band $\mathbf{K} \cdot \mathbf{P}$ model using a graded and asymmetric interface profile. ${ }^{22}$ In the present work, we will implement the $H_{\mathrm{BF}}$ model and investigate the intrinsical optical anisotropy in the InAs/GaSb NCA SL system. To calculate the electronic band structure and associated wave functions, we use an eight-band $\mathbf{K} \cdot \mathbf{P}$ model that incorporates the MIA effect and solve the resulting EFA Hamiltonian equation using the finite difference method. With the obtained eigenvalues and eigenstates obtained, we employ a semiclassic Boltzmann equation approach to calculate the optical properties of the corresponding SL system such as the optical-absorption spectra and polarization spectra. Furthermore, the dependence of the optical polarization on temperature, photoexcited carrier density, and layer width is examined in detail. Our aim is to understand more deeply the physical mechanisms of the optical anisotropy or polarization in the InAs/GaSb NCA SLs.

This paper is organized as follows. In Sec. II, first an eight-band $\mathbf{K} \cdot \mathbf{P}$ model incorporating the MIA effect is developed to calculate the electronic band structure of InAs/

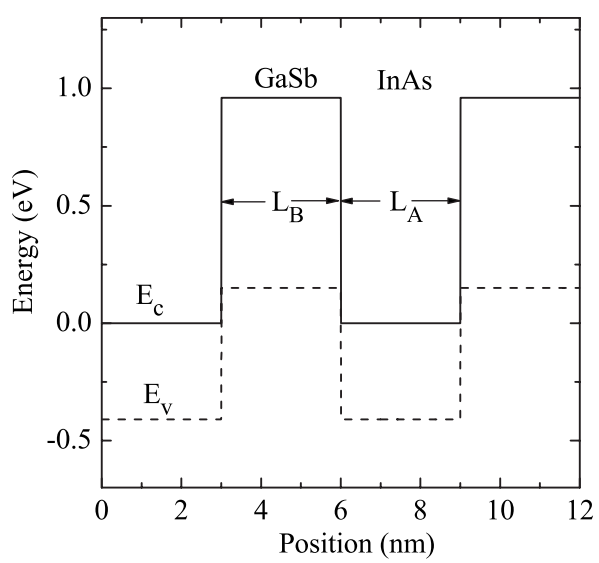

FIG. 1. Illustration of the band alignment for an InAs/GaSb SL grown along the [001] direction ( $z$ axis). Here, $E_{c}$ and $E_{v}$ are, respectively, the unstrained conduction- and valence-band edges, and $L_{A}$ and $L_{B}$ are, respectively, the InAs and GaSb layer widths.

GaSb NCA SLs using the finite difference method and then a semiclassic Boltzmann equation approach is employed to calculate the optical properties of the corresponding SL system. In Sec. III, the numerical results are presented and discussed. Finally, our concluding remarks are summarized in Sec. IV.

\section{THEORETICAL APPROACHES}

Within an eight-band $\mathbf{K} \cdot \mathbf{P}$ formalism, we include the MIA effect for NCA semiconductor SLs grown along [001] direction. By imposing the Bloch boundary conditions on the system, the resulting EFA Hamiltonian equations can be solved for the SL band structure and associated wave functions by the finite difference method. Subsequently, a semiclassic Boltzmann equation approach is employed to calculate the optical properties for the corresponding SL system.

\section{A. Band-structure calculation}

Consider a NCA SL structure constructed by alternating a thin InAs layer with thickness $L_{A}$ and a thin GaSb layer with thickness $L_{B}$ grown along the [001] direction. This growth direction is defined as the $z$ axis. The $x$ and $y$ axes are along [100] and [010], respectively. The band alignment of such an InAs/GaSb SL is shown in Fig. 1. With the set of the following basis states:

$$
|S \uparrow\rangle,|X \uparrow\rangle,|Y \uparrow\rangle,|Z \uparrow\rangle,|S \downarrow\rangle,|X \downarrow\rangle,|Y \downarrow\rangle,|Z \downarrow\rangle,
$$

the eight-band $\mathbf{K} \cdot \mathbf{P}$ Hamiltonian can be written as ${ }^{23}$

$$
H(\mathbf{K})=H_{K}+H_{S}+H_{I},
$$

where $\mathbf{K}=\left(\mathbf{k}, k_{z}\right)=\left(k_{x}, k_{y}, k_{z}\right)$ with $\mathbf{k}$ being the in-plane wave vector, $H_{K}$ is the dominant $\mathbf{K}$-dependent term, $H_{S}$ is the spinorbit interaction term, and the term $H_{I}$ describes the interface related Hamiltonian. The last term $H_{I}$ includes the MIA effect. The $\mathbf{K}$-dependent term $H_{K}$ has the following blockdiagonal form: 


$$
H_{K}=\left[\begin{array}{cc}
H_{4}^{K} & 0 \\
0 & H_{4}^{K}
\end{array}\right] \text {, }
$$

where

$$
H_{4}^{K}=\left[\begin{array}{cccc}
E_{c}+D_{c} & i P k_{x} & i P k_{y} & i P k_{z} \\
-i P k_{x} & E_{v}^{\prime}+D_{x} & N k_{x} k_{y} & N k_{x} k_{z} \\
-i P k_{y} & N k_{x} k_{y} & E_{v}^{\prime}+D_{y} & N k_{y} k_{z} \\
-i P k_{z} & N k_{x} k_{z} & N k_{y} k_{z} & E_{v}^{\prime}+D_{z}
\end{array}\right] .
$$

Here, $\quad D_{c}=A_{c}\left(k_{x}^{2}+k_{y}^{2}+k_{z}^{2}\right), \quad D_{x}=L k_{x}^{2}+M\left(k_{y}^{2}+k_{z}^{2}\right), \quad D_{y}=L k_{y}^{2}$ $+M\left(k_{x}^{2}+k_{z}^{2}\right), D_{z}=L k_{z}^{2}+M\left(k_{x}^{2}+k_{y}^{2}\right)$, and $E_{v}^{\prime}=E_{v}-\Delta / 3 ; E_{c}$ and $E_{v}$ denote, respectively, the unstrained conduction- and valence-band edges; $\Delta$ is the spin-orbit split-off energy; $P$ is the interband momentum matrix element defined as $P$ $=-i\left(\hbar / m_{0}\right)\left\langle S\left|p_{x}\right| X\right\rangle$; and the band parameters $A_{c}, L, M$, and $N$ are given by ${ }^{23}$

$$
\begin{gathered}
A_{c}=\hbar^{2} / 2 m_{e}-2 P^{2} / 3 E_{g}-P^{2} / 3\left(E_{g}+\Delta\right), \\
L=-\left(\hbar^{2} / 2 m_{0}\right)\left(\gamma_{1}+4 \gamma_{2}\right)+P^{2} / E_{g}, \\
M=-\left(\hbar^{2} / 2 m_{0}\right)\left(\gamma_{1}-2 \gamma_{2}\right), \\
N=-\left(\hbar^{2} / 2 m_{0}\right)\left(6 \gamma_{3}\right)+P^{2} / E_{g},
\end{gathered}
$$

where $E_{g}=E_{c}-E_{v}$ is the bulk band gap, $m_{0}, m_{e}$ are the free and effective electron masses, and $\gamma_{i}(i=1,2,3)$ are the Luttinger parameters.

The Hamiltonian due to spin-orbit interaction, $H_{S}$, is given by

$$
H_{S}=-\frac{\Delta}{3}\left[\begin{array}{cccccccc}
0 & 0 & 0 & 0 & 0 & 0 & 0 & 0 \\
0 & 0 & i & 0 & 0 & 0 & 0 & -1 \\
0 & -i & 0 & 0 & 0 & 0 & 0 & i \\
0 & 0 & 0 & 0 & 0 & 1 & -i & 0 \\
0 & 0 & 0 & 0 & 0 & 0 & 0 & 0 \\
0 & 0 & 0 & 1 & 0 & 0 & -i & 0 \\
0 & 0 & 0 & i & 0 & i & 0 & 0 \\
0 & -1 & -i & 0 & 0 & 0 & 0 & 0
\end{array}\right] .
$$

The MIA-related term $H_{I}$ has the following blockdiagonal form:

$$
H_{I}=\left[\begin{array}{cc}
H_{4}^{I} & 0 \\
0 & H_{4}^{I}
\end{array}\right]
$$

where the expression of the microscopic interface Hamiltonian $H_{4}^{I}$ with respect to the basis states $|S\rangle,|X\rangle,|Y\rangle$, and $|Z\rangle$ can be found in Ref. 4 and has the form

$$
H_{4}^{I}=H_{X Y}\left[\begin{array}{llll}
0 & 0 & 0 & 0 \\
0 & 1 & 1 & 0 \\
0 & 1 & 1 & 0 \\
0 & 0 & 0 & 1
\end{array}\right],
$$

where $H_{X Y}$ is an adjustable parameter characterizing the strength of the interface potential. This $4 \times 4$ matrix takes into account the MIA-induced coupling between one $s$-like conduction-band state $(|S\rangle)$ and three $p$-like valence-band states $(|X\rangle,|Y\rangle$, and $|Z\rangle)$. Because of the interface symmetry the interface potential does not directly couple the conduction-band states to the valence-band states. ${ }^{24}$ Hence, one may anticipate that the conduction bands will be less affected by the MIA effect than the valence bands in the NCA SLs. The interface potential is short range and is localized at the SL interface within about half a monolayer ( $\sim 2 \AA$ ).

In the present model, we neglect (i) bulk inversion asymmetry terms resulting from the lack of inversion symmetry in zinc-blende semiconductors because the MIA effect dominates in our short-period InAs/GaSb SL structures, ${ }^{13}$ and (ii) the strain effect due to the small lattice mismatch between InAs and GaSb layers $(\sim 0.39 \%)$.

With the EFA theory, we solve for the SL band structure by transforming the eight-band $\mathbf{K} \cdot \mathbf{P}$ Hamiltonian into a set of eight coupled second-order differential equations by replacing $k_{z}$ with $-i d / d z$. Products of $k_{z}$ with positiondependent material parameters (quadratic polynomials in $k_{z}$ ) are symmetrized ${ }^{17,18}$ before converting the equations to differential form and the set of differential equations so formulated is then Hermitian. The energy dispersions and the corresponding wave functions for the SL are derived from the Schrödinger equation

$$
H \Psi=E \Psi,
$$

where $\Psi$ is the multicomponent wave function and $E$ the corresponding energy. Usually the SL wave function $\Psi_{n}(\mathbf{k}, q, \mathbf{r}, z)$ can be expanded as a linear combination of the basis states $U_{\nu}(\mathbf{r})$ and the envelope function $F_{\nu}(n, \mathbf{k}, q, z)$, which reads

$$
\Psi_{n}(\mathbf{k}, q, \mathbf{r}, z)=e^{i \mathbf{k} \cdot \mathbf{r}} \sum_{\nu=1}^{8} U_{\nu}(\mathbf{r}) F_{\nu}(n, \mathbf{k}, q, z),
$$

where $\mathbf{r}=(x, y)$ is the in-plane position vector, $n(\nu)$ is the subband (basis) index, $q$ is the SL wave vector along the growth direction ( $z$ axis), $U_{\nu}(\mathbf{r})$ is the zone-center basis state described by Eq. (1), and $F_{\nu}(n, \mathbf{k}, q, z)$ is the $\nu$ th component of the $n$th subband envelope function along the $z$ axis. By replacing $k_{z}$ with $-i d / d z$ in the $\mathbf{K} \cdot \mathbf{P}$ Hamiltonian, the resulting eight-band envelope function equation can be written as

$$
\sum_{\mu=1}^{8} H_{\mu \nu}(\mathbf{k},-i d / d z) F_{\mu}(n, \mathbf{k}, q, z)=E_{n}(\mathbf{k}, q) F_{\nu}(n, \mathbf{k}, q, z) .
$$

Using the finite difference technique and imposing the Bloch boundary conditions ${ }^{25}$ one can reduce Eq. (11) to a single eigenvalue equation of the form $H \cdot \mathbf{F}=E \cdot \mathbf{F}$, where the Hamiltonian matrix can be diagonalized by standard mathematical subroutines to obtain the eigenvalues $E$ and the eigenvectors $\mathbf{F}$. An important advantage of the finite difference method is that it is quite easy to implement boundary conditions and arbitrary potentials. Additionally, this method is also able to describe the tunneling effect with only a few changes and is numerically stable in contrast to the transfer- 
matrix method ${ }^{26,27}$ which sometimes fails because of growing exponentials.

\section{B. Optical-absorption coefficient}

After obtaining the band structure and the wave functions by solving the EFA Hamiltonian equations described above, the next step is to calculate the optical-matrix element, because the strength of the optical absorption is proportional to the square of the optical-matrix element. We denote, respectively, the initial (valence-band) and final (conduction-band) states involved in the interband optical transition process with $\Psi_{n}(\mathbf{k}, q, \mathbf{r}, z)$ and $\Psi_{n^{\prime}}(\mathbf{k}, q, \mathbf{r}, z)$. For a given light polarization direction $\boldsymbol{\epsilon}$, the optical-matrix element between such two states can be written as

$$
\begin{aligned}
\boldsymbol{\epsilon} \cdot \mathbf{M}_{n n^{\prime}}(\mathbf{k}, q)= & \left\langle\Psi_{n^{\prime}}(\mathbf{k}, q, \mathbf{r}, z)|\boldsymbol{\epsilon} \cdot \mathbf{p}| \Psi_{n}(\mathbf{k}, q, \mathbf{r}, z)\right\rangle \\
= & \boldsymbol{\epsilon} \cdot \sum_{\mu \nu}\left(\left\langle F_{\nu}\left(n^{\prime}, \mathbf{k}, q, z\right) \mid F_{\mu}(n, \mathbf{k}, q, z)\right\rangle\right. \\
& \times\left\langle U_{\nu}(\mathbf{r})|\mathbf{p}| U_{\mu}(\mathbf{r})\right\rangle \\
& \left.+\left\langle F_{\nu}\left(n^{\prime}, \mathbf{k}, q, z\right)|\mathbf{p}| F_{\mu}(n, \mathbf{k}, q, z)\right\rangle \delta_{\mu \nu}\right),
\end{aligned}
$$

where the first term gives the interband optical transition strength and the second term, which gives the strength of the intraband optical transition, involves essentially the overlap between the same envelope function components for initial and final states. Thus, one can anticipate that it also contributes to the interband optical transition strength in InAs/GaSb SL systems, where the conduction-band states are strongly mixed with the valence-band states because of the type-II band alignment at the InAs/GaSb interfaces.

Inserting the optical-matrix element given by Eq. (12) into Fermi's golden rule, the electronic transition rate induced by direct carrier-photon interaction via absorption scattering can be obtained as

$$
\begin{aligned}
W_{n n^{\prime}}(\mathbf{k}, q)= & \frac{2 \pi}{\hbar}\left(\frac{e F_{0}}{m_{0} \omega}\right)^{2}\left|\boldsymbol{\epsilon} \cdot \mathbf{M}_{n n^{\prime}}(\mathbf{k}, q)\right|^{2} \delta\left[E_{n^{\prime}}(\mathbf{k}, q)\right. \\
& \left.-E_{n}(\mathbf{k}, q)-\hbar \omega\right],
\end{aligned}
$$

where $F_{0}$ and $\omega$ are, respectively, the strength and frequency of the light field.

In this study, we employ the semiclassic Boltzmann equation as the governing transport equation to study the photo response of a SL to the applied light field. In case of degenerate statistics, the Boltzmann equation takes the form

$$
\frac{\partial f_{n^{\prime}}(\mathbf{k}, q, t)}{\partial t}=\sum_{n} \sum_{\mathbf{k}, q}\left[F_{n n^{\prime}}(\mathbf{k}, q, t)-F_{n^{\prime} n}(\mathbf{k}, q, t)\right] .
$$

Here, $\quad F_{n n^{\prime}}(\mathbf{k}, q, t)=f_{n}(\mathbf{k}, q, t)\left[1-f_{n^{\prime}}(\mathbf{k}, q, t)\right] W_{n n^{\prime}}(\mathbf{k}, q)$, $f_{n}(\mathbf{k}, q, t)$ is the momentum distribution function for the electron in a state $|n, \mathbf{k}, q\rangle$, where $\langle\mathbf{R} \mid n, \mathbf{k}, q\rangle=\Psi_{n}(\mathbf{k}, q, \mathbf{R})$ and $W_{n n^{\prime}}(\mathbf{k}, q)$ is the steady-state electronic transition rate. In Eq. (14), the effect of the light field has been included within the time-dependent momentum distribution functions and within the electronic transition rates. Thus, to avoid double count- ing, the force term induced by the light field does not appear on the left-hand side of the Boltzmann equation. It is known that there is no simple and analytical solution for Eq. (14) with the electronic transition rate given by Eq. (13). In this work, we apply the usual energy balance-equation approach to solve the Boltzmann equation approximately. ${ }^{28,29}$ This balance equation can be derived by multiplying $\Sigma_{n^{\prime}} \Sigma_{\mathbf{k}, q} E_{n^{\prime}}(\mathbf{k}, q)$ on both sides of Eq. (14), which reads

$$
P_{t}=\hbar \omega \sum_{n, n^{\prime}} \sum_{\mathbf{k}, q} f_{n}(\mathbf{k}, q, t)\left[1-f_{n^{\prime}}(\mathbf{k}, q, t)\right] W_{n n^{\prime}}(\mathbf{k}, q),
$$

where $P_{t}=\partial\left[\sum_{n^{\prime}} \Sigma_{\mathbf{k}, q} E_{n^{\prime}}(\mathbf{k}, q) f_{n^{\prime}}(\mathbf{k}, q, t)\right] / \partial t$ is the electronic energy-transfer rate per cell of the SL. The optical-absorption coefficient can be calculated through ${ }^{30}$

$$
\alpha=\alpha_{0}\left(2 \hbar P_{t} / e^{2} F_{0}^{2}\right),
$$

where $\alpha_{0}=e^{2} /\left(\hbar \epsilon_{0} c \sqrt{\kappa}\right), \kappa$, and $\epsilon_{0}$ are, respectively, the dielectric constants of the material layer and the free space, and $c$ is the velocity of light in vacuum. Thus, the opticalabsorption coefficient is obtained as

$$
\begin{aligned}
\alpha= & C \sum_{n, n^{\prime}} \sum_{\mathbf{k}, q} f\left[E_{n}(\mathbf{k}, q)\right]\left\{1-f\left[E_{n^{\prime}}(\mathbf{k}, q)\right]\right\}\left|\boldsymbol{\epsilon} \cdot \mathbf{M}_{n n^{\prime}}(\mathbf{k}, q)\right|^{2} \\
& \times \delta\left[E_{n^{\prime}}(\mathbf{k}, q)-E_{n}(\mathbf{k}, q)-\hbar \omega\right],
\end{aligned}
$$

where $C=4 \pi \hbar \alpha_{0} /\left(m_{0}^{2} \omega\right)$. Here we used a steady-state energy distribution function such as the Fermi-Dirac function for the distribution function, i.e., $f_{n}(\mathbf{k}, q, t) \simeq f\left[E_{n}(\mathbf{k}, q)\right]$. The summation in Eq. (17) has to be evaluated numerically.

\section{NUMERICAL RESULTS AND DISCUSSIONS}

The input material parameters for InAs and GaSb used in the present study are taken from Ref. 25. The overlap energy between the InAs conduction band and the $\mathrm{GaSb}$ valence band is taken to be $150 \mathrm{meV}$. It is known that in NCA InAs/ GaSb SLs, there are two different kinds of interfaces: the BA interface GaSb-on-InAs at one side which can be grown InSb-like and the $\mathrm{AB}$ interface InAs-on-GaSb at the other side which can be grown GaAs like. ${ }^{31}$ Thus, one has two additional parameters $H_{X Y}^{B A}$ and $H_{X Y}^{A B}$ characterizing the strength of the InSb-like and GaAs-like interface potentials, which need to be determined in the calculation. Fortunately, we can obtain these two parameters by fitting the experimental data. We allow $H_{X Y}^{A B}$ and $H_{X Y}^{B A}$ to vary such that it reaches the best agreement between the experimental and theoretical band gaps as shown in Fig. 2, where in the upper panel the InAs width $L_{A}$ is varied while the GaSb width $L_{B}$ is held constant and in the lower panel the GaSb width $L_{B}$ is varied while the InAs width $L_{A}$ is held constant. The best agreement was obtained for $H_{X Y}^{A B}=870 \mathrm{meV}$ and $H_{X Y}^{B A}=490 \mathrm{meV}$ which are of the same magnitude as those used in previous works. ${ }^{12,13} H_{X Y}^{A B} \neq H_{X Y}^{B A}$ is due to the fact that the two interfaces are not identical.

We use a test InAs/GaSb NCA SL to display the main results of our band-structure calculation. The InAs/GaSb layer widths of the test structure are $L_{A}\left(L_{B}\right)=25(25) \AA$. The band structures of this SL calculated without and with the 

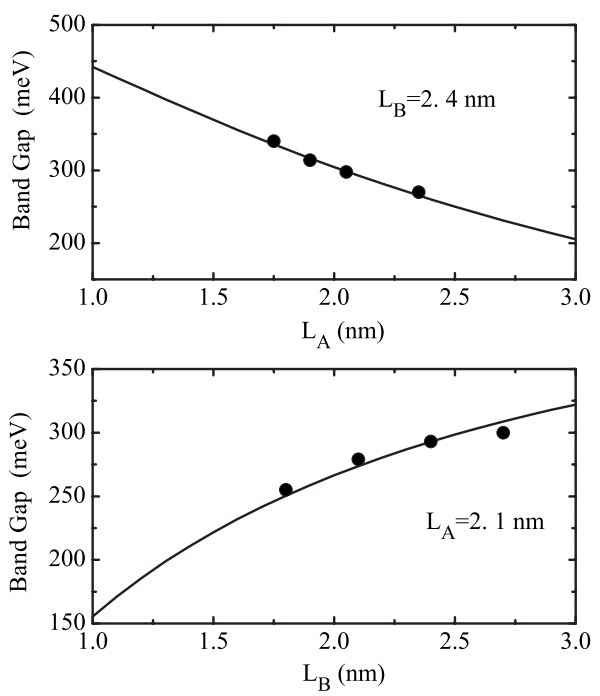

FIG. 2. Band-gap energy as a function of InAs (GaSb) width $L_{A}$ $\left(L_{B}\right)$ for a fixed GaSb (InAs) width $L_{B}\left(L_{A}\right)$ as indicated. Solid circles are the experimental data (Ref. 32) and solid line represents our theoretical results.

MIA effect are shown in Figs. 3(a) and 3(b), respectively, where $d=L_{A}+L_{B}$ is the SL period. There are only four energy bands, labeled as CB1 for the first conduction band, HH1 and $\mathrm{HH} 2$ for the first and second heavy-hole bands, and LH1 for the first light-hole band. The assignment of the carrier type to the various bands follows from the associated properties of the envelope function at the zone center. Comparing Figs. 3(a) and 3(b), we notice three major differences: (i) at nonzero in-plane wave vector $\mathbf{k}$ (\| [100] direction), the MIA effect creates a sizable spin slitting for both conduction and valence bands due to the reduced interface symmetry. However, the splitting is clearly more pronounced in the valence bands than in the conduction band. This is because the conduction band is less affected by the MIA effect than the valence bands, which is manifested by the interface Hamiltonian (8) where no direct coupling of conduction band to valence band exists. It appears that the $\mathbf{K} \cdot \mathbf{P}$ matrix elements which couple the electron and the light-hole states play a key role in the splitting of the conduction band; (ii) along the $q$ direction (|| [001] direction), the MIA effect causes an anticrossing behavior between the LH1 and HH2 minibands which occurs at nonzero $q$ value, which is consistent with a previous tight-binding calculation. ${ }^{16}$ This anticrossing is due to the zone center HH-LH mixing effect induced by the interface asymmetry; (iii) at the zone center, the MIA effect results in a significant reduction in the band gap. This is because a number of valence-band anticrossings induced by the zone center HH-LH mixing lead to band repulsions, i.e., a rise of the $\mathrm{HH} 1$ band and thus a smaller band gap.

The squared envelope functions at the zone center for the same test SL are calculated without and with the MIA effect and are, respectively, shown in Figs. 4(a) and 4(b). A common feature in Figs. 4(a) and 4(b) is that, because of the different effective masses and different quantum size effects, the heavy holes are largely confined in the GaSb layer whereas the electron (light-hole) envelope functions overlap considerably from one InAs (GaSb) layer to another. When
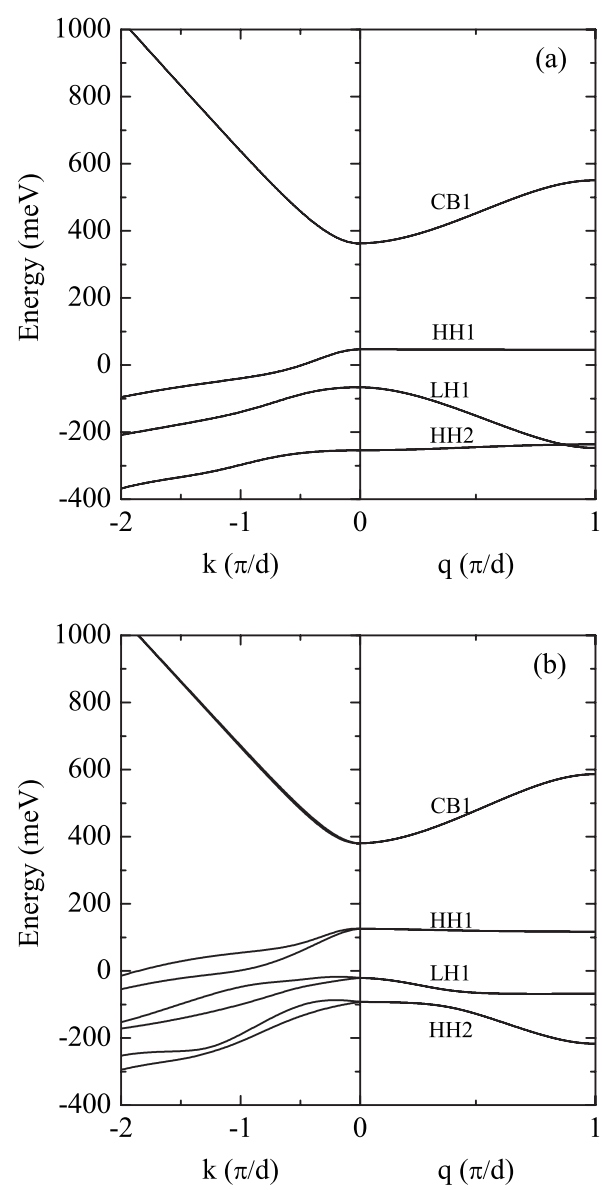

FIG. 3. Band structures of a test InAs/GaSb SL with layer widths $L_{A}=L_{B}=25 \AA$ : (a) calculated without the MIA effect and (b) calculated with the MIA effect.

comparing the panels (a) and (b) in this figure, one can see that the CB1, HH1, LH1, and HH2 envelope functions have peaks at the interface due to the localized interface potential, and what's more, the peaks in the $\mathrm{HH} 1$ and $\mathrm{HH} 2$ envelope functions are much sharper than those of the CB1 and LH1 envelope functions because the heavy holes are more bounded by the interface potential due to their larger effective masses. The sharp peaks of the $\mathrm{HH}$ envelope functions indicate that the interface potential has an ability to bind the hole at the interface. More importantly, due to the above features of the envelope functions, the overlap between the electron and the hole states is expected to be considerably enhanced by the MIA effect, which implies a strong type-II optical transition in the InAs/GaSb NCA SL system.

For a given light polarization direction $\boldsymbol{\epsilon}$, we first define the squared optical matrix element for the transition between the bands $\zeta$ and $\eta$,

$$
I_{\zeta \eta}(\boldsymbol{\epsilon}, \mathbf{k}, q)=\sum_{n n^{\prime}}\left|\boldsymbol{\epsilon} \cdot \mathbf{M}_{n n^{\prime}}(\mathbf{k}, q)\right|^{2},
$$

where $n$ runs over the states in the $\zeta$ band and $n^{\prime}$ runs over the states in the $\eta$ band. Then, the optical anisotropy with respect to the light polarization directions $\boldsymbol{\epsilon}_{1}$ and $\boldsymbol{\epsilon}_{2}$ can be evaluated by the polarization ratio 

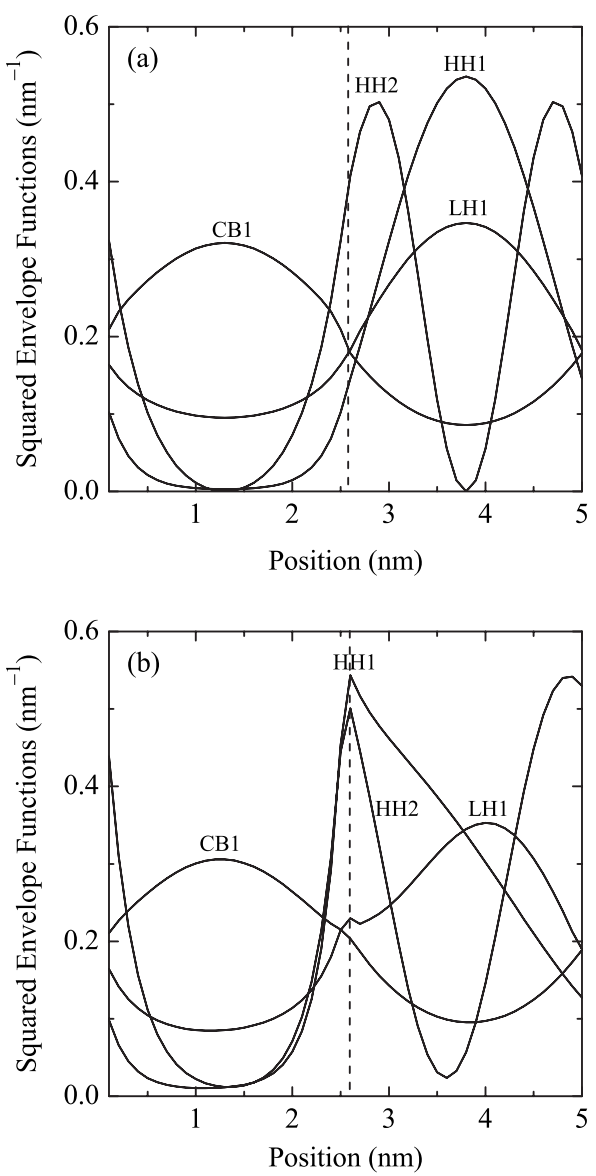

FIG. 4. Squared envelope functions at the zone center for the test InAs/GaSb SL with $L_{A}=L_{B}=25 \AA$ : (a) calculated without the MIA effect and (b) calculated with the MIA effect. The vertical dashed lines indicate the interface between InAs and GaSb.

$$
\lambda_{\zeta \eta}(\mathbf{k}, q)=\frac{\left|I_{\zeta \eta}\left(\boldsymbol{\epsilon}_{2}, \mathbf{k}, q\right)-I_{\zeta \eta}\left(\boldsymbol{\epsilon}_{1}, \mathbf{k}, q\right)\right|}{\left|I_{\zeta \eta}\left(\boldsymbol{\epsilon}_{2}, \mathbf{k}, q\right)+I_{\zeta \eta}\left(\boldsymbol{\epsilon}_{1}, \mathbf{k}, q\right)\right|} .
$$

It was shown previously that the MIA effect due to the low $C_{2 v}$ point-group symmetry causes an optical anisotropy with respect to the light polarization direction in the NCA SLs. ${ }^{5-7}$ To observe a pronounced optical anisotropy, the light polarization direction is normally set as the $[110]$ and $[1 \overline{1} 0]$ directions in the experiment, ${ }^{5,6}$ which will become clear in the following. In our numerical calculation, we vary $\boldsymbol{\epsilon}$ over all the in-plane polarization angles $\theta(0 \sim 2 \pi)$ and calculate the squared optical matrix elements for the test InAs/GaSb SL as a function of $\theta$. Figures 5(a) and 5(b) display, respectively, the calculated results for the HH1-CB1 and LH1-CB1 transitions at the zone center without and with the MIA effect. Comparing panels (a) and (b) in this figure, we notice that (i) in the absence of the MIA effect, the optical matrix elements are independent of the light polarization direction, which is manifested by the isotropic circles in panel (a); and (ii) with the MIA effect included, the optical matrix elements exhibit a strong in-plane anisotropy and have a twofold in-plane symmetry which corresponds to the $C_{2 v}$ point-group symmetry of the NCA SL system [see panel (b)]. It is clear that the
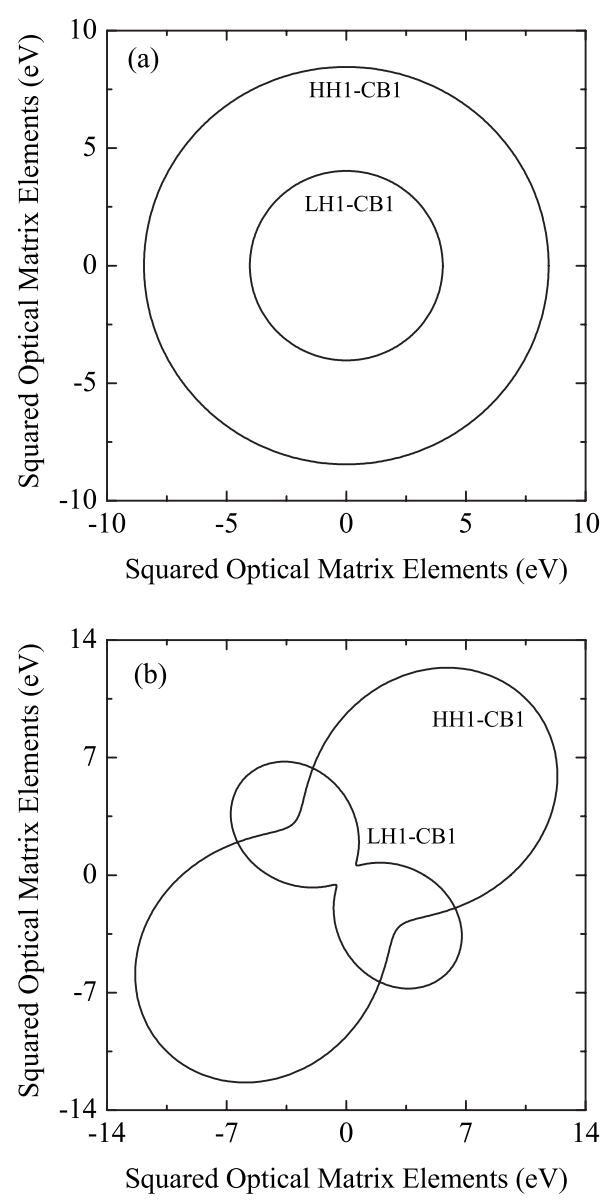

FIG. 5. Contour plot of angular dependence of the squared optical matrix elements at the zone center for the test InAs/GaSb SL: (a) calculated without the MIA effect and (b) calculated with the MIA effect.

optical anisotropy is induced by the MIA effect and the largest anisotropy is induced by the light polarized along the $[110]$ and $[1 \overline{10}]$ directions. The reason why this gives the largest optical anisotropy is the nonequivalence of the [110] and $[1 \overline{1} 0]$ in-plane directions at two successive interface planes where the chemical bonds are spatially arranged differently. From the device application point of view, our results provide guidelines for designing optical devices which require the largest optical polarization to achieve an optimal performance. In the following, when calculating the intrinsic optical anisotropy in the InAs/GaSb NCA SLs, we will set the light polarized along the [110] and [1피 directions as in the experiments ${ }^{5,6}$ because these two directions give rise to the largest optical anisotropy.

The optical-matrix elements of the HH1-CB1, LH1-CB1, and HH2-CB1 transitions for the test InAs/GaSb SL as function of the in-plane wave vector $\mathbf{k}$ and the wave vector $q$ along the growth direction are shown in Fig. 6, where panels (a) and (b) are, respectively, calculated without and with the MIA effect. As can be seen from Figs. 6(a) and 6(b): (i) since the test SL considered here is thin $\left(L_{A}=L_{B}=25 \AA\right)$, the optical matrix elements are strongly dependent on both $\mathbf{k}$ and $q$; and (ii) along both the $\mathbf{k}$ and the $q$ directions, the MIA effect 

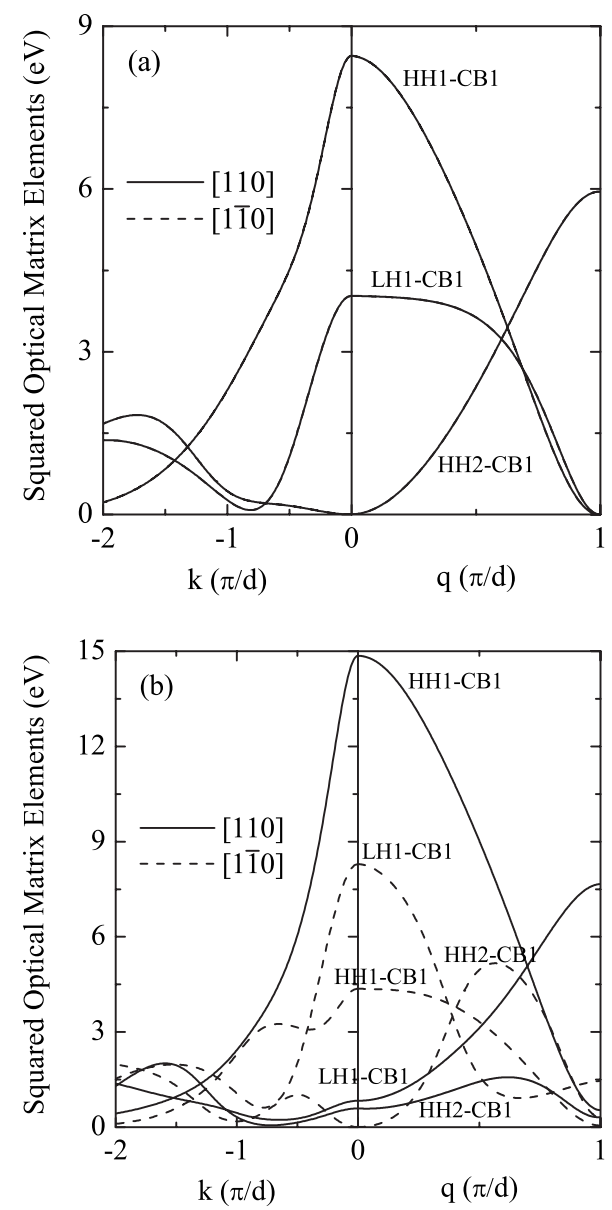

FIG. 6. Squared optical matrix elements for the test InAs/GaSb as functions of the in-plane wave vector $\mathbf{k}$ and the growth wave vector $q$ : (a) calculated without the MIA effect and (b) calculated with the MIA effect. The solid and dashed lines represent, respectively, the [110] and [1 $\overline{1} 0]$ light polarization directions.

gives rise to a large optical anisotropy in the optical-matrix elements induced by [110]- and [1 $1 \overline{1} 0]$-polarized light. This is attributed to the fact that the MIA effect breaks not only the rotational symmetry around the $z$ axis or in-plane symmetry which causes the k-dependent anisotropy in the opticalmatrix elements but also the translational invariance along the $z$ axis or the growth symmetry that produces the $q$-dependent anisotropy in the optical-matrix elements.

To calculate the optical-absorption coefficient for the NCA InAs/GaSb SL system, we use a Lorentzian function to broaden the energy conservation delta function in Eq. (17). Namely, we take $\delta(x)=(\Gamma / \pi) /\left(x^{2}+\Gamma^{2}\right)$ with $\Gamma$ being the broadening factor induced by different scattering mechanisms. Furthermore, we use a phenomenological formula ${ }^{33}$ to calculate this broadening factor: $\Gamma=\Gamma_{1}+\Gamma_{2} /\left[\exp \left(\hbar \omega_{0} / k_{B} T\right)\right.$ -1] with $\Gamma_{1}$ and $\Gamma_{2}$ being constants and $\omega_{0}$ the opticalphonon frequency. The first and the second term in this formula represent, respectively, the broadening factors induced by temperature-independent and temperature-dependent scattering mechanisms, i.e., time-independent scattering mechanisms mainly refer to the scattering of carriers with impurity and interface roughness and those of temperature-dependent
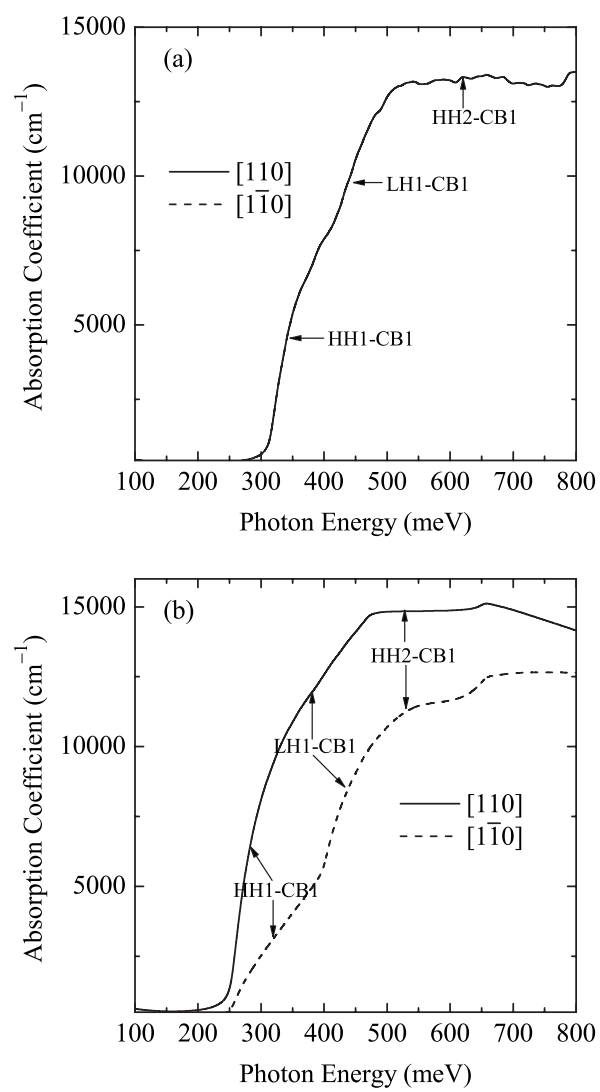

FIG. 7. Optical absorption spectra for the test InAs/GaSb SL: (a) calculated without the MIA effect and (b) calculated with the MIA effect. The results are calculated at a fixed temperature $T=77 \mathrm{~K}$ and a fixed photoexcited carrier density $N_{0}=10^{16} \mathrm{~cm}^{-3}$. The solid and dashed lines represent, respectively, the [110] and [1 $\overline{1} 0]$ light polarization directions.

refer to the scattering of carriers with acoustic-phonons and optical-phonons. As the optical-phonon frequencies for InAs and $\mathrm{GaSb}$ is nearly equal, we take $\hbar \omega_{0}(\mathrm{InAs}) \simeq \hbar \omega_{0}(\mathrm{GaSb})$ $=30 \mathrm{meV}$. Because there are no published values for $\Gamma_{1}$ and $\Gamma_{2}$ in the InAs/GaSb SL system, in the present work we take $\Gamma_{1}=4.5 \mathrm{meV}$ and $\Gamma_{2}=44.3 \mathrm{meV}$. With such two values, the broadening factor $\Gamma$ is $5 \mathrm{meV}$ at $T=77 \mathrm{~K}$ and $25 \mathrm{meV}$ at $T=300 \mathrm{~K}$, which is in line with the magnitude of general temperature-dependent absorption linewidths. ${ }^{34,35}$ It is known that in an undoped InAs/GaSb SL, the presence of the light field can pump electrons from the GaSb valance band into the InAs conduction band. In general, the density of photoexcited carriers depends on the light intensity and frequency as well as other scattering and relaxation mechanisms. In this study, we assume that the photoexcited carrier density $N_{0}$ in undoped SLs is about $10^{15}-10^{18} \mathrm{~cm}^{-3}$.

Figures 7(a) and 7(b) show the optical-absorption spectra for the test InAs/GaSb SL calculated without and with the MIA effect, respectively, for a fixed temperature $(T=77 \mathrm{~K})$ and a fixed photoexcited carrier density $\left(N_{0}=10^{16} \mathrm{~cm}^{-3}\right)$. The optical-absorption spectra are found to be quite broad and nearly structureless. This is because the thin SL considered here has a strong dispersed subband and miniband structure along the $\mathbf{k}$ and the $q$ directions. Due to the lifting of the parity selection rules in the type-II NCA SL system, the 


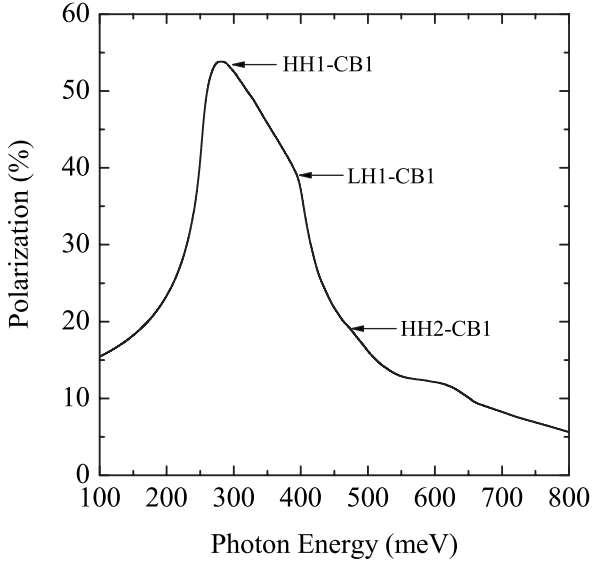

FIG. 8. Relative difference or polarization ratio for Fig. 7(b). The temperature is fixed at $T=77 \mathrm{~K}$ and the photoexcited carrier density is fixed at $N_{0}=10^{16} \mathrm{~cm}^{-3}$.

parity-forbidden optical transition between the $\mathrm{HH} 2$ and $\mathrm{CB} 1$ states with opposite parity becomes allowed, which is not so in type-I CA SL structures, where only the parity-allowed optical transitions between the $\mathrm{HH}(\mathrm{LH})$ and the $\mathrm{CB}$ states with the same parities such as the HH1-CB1 and LH1-CB1 transitions can take place. However, the strength of the HH2$\mathrm{CB} 1$ optical transition is small due to the very small overlap between the $\mathrm{HH} 2$ and the $\mathrm{CB} 1$ envelope functions at the interface. Comparing Figs. 7(a) and 7(b), we notice that the MIA effect causes a giant in-plane optical anisotropy in the optical-absorption coefficients for [110]- and [1 $\overline{1} 0]$-polarized light, which is a direct consequence of the polarized optical matrix elements shown in Fig. 6(b). Because the absorption strength is proportional to the optical matrix elements, it is larger in the presence of the MIA effect than in the absence of this effect for [110]-polarized light and the case is reversed for [1 $1 \overline{1} 0]$-polarized light. In addition, the absorption cutoff is blueshifted due to the MIA effect because this effect reduces the fundamental band gap.

The relative difference or polarization ratio between the two polarized absorption spectra shown in Fig. 7(b) is plotted as a function of photon frequency in Fig. 8. From this figure, we can see that: (i) the polarization spectrum is quite broad (over the whole spectral region) since all the transitions from the valence bands $(\mathrm{HH} 1, \mathrm{HH} 2$, and $\mathrm{LH} 1)$ to the conduction band (CB1) contribute to the optical absorption; and (ii) the maximum value of the polarization ratio is close to $55 \%$ and the position of this value is located within the spectral region for the HH1-CB1 transitions. This is because the largest relative difference between the two polarized optical matrix elements, as shown in Fig. 6(b), occurs for the HH1-CB1 transitions. Thus the highest polarization ratio can be observed within the spectral region for these transitions.

To demonstrate that the optical anisotropy is more sensitive to the MIA effect in type-II NCA SLs than in type-I NCA SLs, we compared our results for the InAs/GaSb system with those obtained by Krebs et al. ${ }^{5}$ for the GaAs/InP system. The polarization spectra in those two systems are remarkably different, which is manifested by the maximum value of the polarization ratio (up to $55 \%$ vs up to $40 \%$ ) and

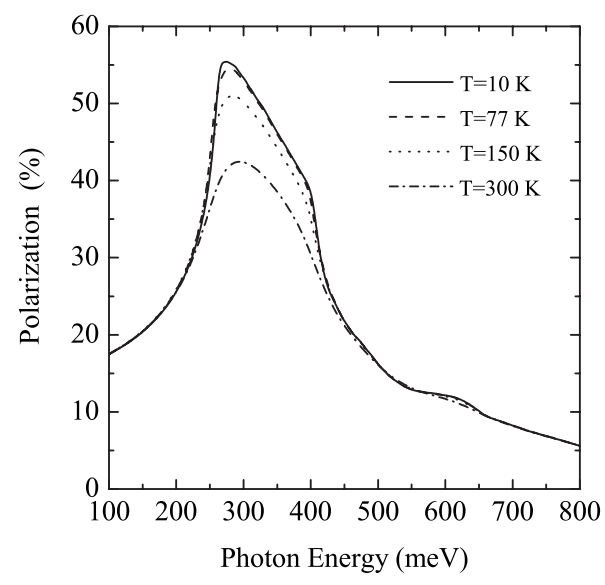

FIG. 9. Dependence of the polarization ratio for the test InAs/ $\mathrm{GaSb} \mathrm{SL}$ on the temperature as indicated. The photoexcited carrier density is fixed at $N_{0}=10^{16} \mathrm{~cm}^{-3}$.

by the broadening of the polarization spectrum (about 350 $\mathrm{meV}$ vs about $50 \mathrm{meV}$ ). Thus, the optical anisotropy in InAs/ GaSb NCA SLs is more sensitive to the MIA effect because it depends essentially on the overlap between the electron and the hole wave functions at the interface.

Figure 9 displays the temperature dependence of the polarization spectrum for the test InAs/GaSb SL as a function of photon frequency. The photoexcited carrier density is fixed at $N_{0}=10^{16} \mathrm{~cm}^{-3}$. As can be seen from this figure, the polarization ratio decreases and the spectral shape broadens with increasing temperature. The polarization ratio is strongly temperature dependent at high temperatures ( $T$ $>77 \mathrm{~K}$ ) because for this temperature region the main contribution to the broadening factor $\Gamma$ comes from the scattering of carriers with optic phonons while it is nearly temperature independent at low temperatures $(T<77 \mathrm{~K})$ since at this temperature region the broadening is dominated by the scattering of carrier with interface roughness (we assume an impurity-free SL structure). Thus, we anticipate that the scattering mechanisms will greatly affect the polarization spectrum induced by the MIA effect in the NCA SL system.

The dependence of the polarization spectrum on the photoexcited carrier density for the test InAs/GaSb is plotted as a function of the photon frequency in Fig. 10. The temperature is fixed at $T=77 \mathrm{~K}$. From this figure, we notice that both the peak position and magnitude of the polarization spectrum vary significantly with the photoexcited carrier density. With increasing density, the quasi-Fermi levels for electrons in the InAs layer and holes in the GaSb layer rises and falls, respectively. As a consequence, the peak position of the polarization spectrum is blueshifted because it reflects the energy separation between the electron and hole quasiFermi levels around which the strongest optical transitions occur. In addition, the magnitude of the peak in the polarization spectrum decreases with increasing photoexcited carrier density. The reason is that a higher density opens up a smaller number of optical transition channels due to band filling effects and thus induces a smaller polarization ratio as derived from the corresponding polarized optical-absorption coefficients. Therefore, to achieve a larger polarization ratio for InAs/GaSb NCA SLs, it is necessary to work at relatively low temperatures and low carrier density. 


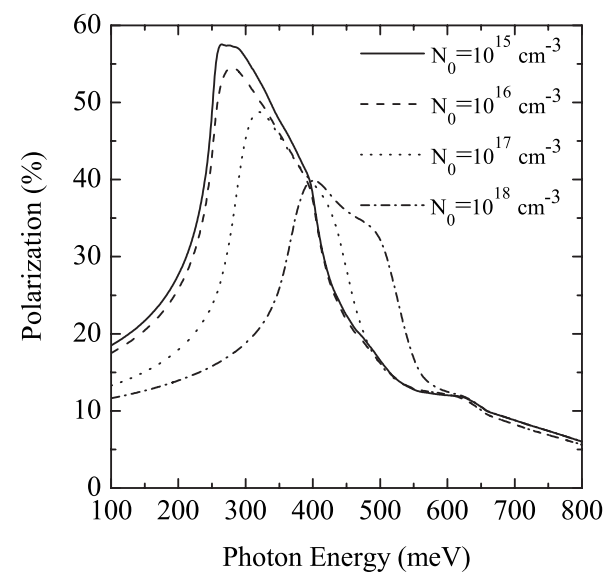

FIG. 10. Dependence of the polarization rate for the test InAs/ $\mathrm{GaSb} \mathrm{SL}$ on the photoexcited carrier density as indicated. The temperature is fixed at $T=77 \mathrm{~K}$.

*An important feature of InAs/GaSb SLs is that the absorption cutoff and strength can be tuned by simply adjusting the InAs and/or GaSb layer widths. Thus, one can anticipate that this feature may have an influence on the opticalabsorption spectrum and thus the polarization spectrum. Fig. 11 shows the polarization spectrum for different InAs/GaSb layer widths at a fixed temperature $T=77 \mathrm{~K}$ and a fixed photoexcited carrier density $N_{0}=10^{16} \mathrm{~cm}^{-3}$. As mentioned above, the peak position of the polarization spectrum is located within the $\mathrm{HH} 1-\mathrm{CB} 1$ transition energies. As a result, it is redshifted when increasing InAs/GaSb layer widths because such an increase reduces the energy separation between the electron and the hole confinement energies due to quantum size effect. Due to the fact that in shorter SL structures the percentage of the interface contribution to the optical anisotropy induced by the MIA effect increases significantly, the peak magnitude of the polarization spectrum increases with decreasing InAs/GaSb layer widths. In addition, one can observe that in the thicker SL structures (i.e., $L_{A}=L_{B}=30,35 \AA$ ), there are other small peaks in the polarization spectra, which result from optical transitions with higher transition energies.

To close this section, we state that the MIA effect on the optical anisotropy in InAs/GaSb NCA SLs as suggested by our theoretical results can be detected in an experiment by measuring the optical-absorption coefficient with respect to the in-plane light polarization direction. The changing of the light polarization direction can be realized by simply using an optical polarizer.

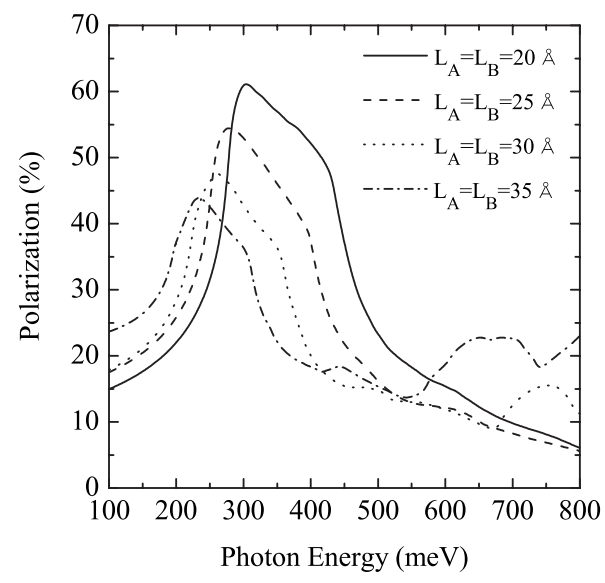

FIG. 11. Polarization spectrum for different InAs/GaSb layer widths as indicated. The temperature is fixed at $T=77 \mathrm{~K}$ and the photoexcited carrier density is fixed at $N_{0}=10^{16} \mathrm{~cm}^{-3}$.

\section{CONCLUSIONS}

We have studied theoretically the intrinsic optical anisotropy induced by the MIA effect as is present in InAs/GaSb NCA SLs. The eight-band $\mathbf{K} \cdot \mathbf{P}$ model incorporating the MIA effect was solved using the finite difference method which gave us the electronic states in such SLs. With the obtained band structure and associated wave functions, we used the Boltzmann equation approach to calculate the optical properties for the corresponding SL system. It was shown that in the InAs/GaSb NCA SL system, the MIA effect causes a large optical anisotropy for linearly polarized light and the largest anisotropy occurs for the [110] and [1 $\overline{10}]$ light polarization directions. The giant optical anisotropy was manifested by the optical matrix elements and the opticalabsorption coefficients. Moreover, we found that the optical anisotropy and the polarization ratio decreases with increasing temperature, photoexcited carrier density and layer widths of InAs/GaSb NCA SLs. These results could enable us to better understand the physical mechanisms behind the application of InAs/GaSb NCA SLs as optical devices which require polarization control and selectivity.

\section{ACKNOWLEDGMENTS}

This work was supported partly by the Flemish Science Foundation (FWO-VL), the Belgium Science Policy (IAP), the NSF of China (Grants No. 10664006, No. 10504036, and No. 90503005), Special Funds of 973 Project of China (Grant No. 2005CB623603), and Knowledge Innovation Program of the Chinese Academy of Sciences.

\footnotetext{
*wenxu_issp@yahoo.cn

†francois.peeters@ua.ac.be

${ }^{1}$ J. H. Ser, Y. G. Ju, J. H. Shin, and Y. H. Lee, Appl. Phys. Lett. 66, 2769 (1995).

${ }^{2}$ Y. G. Ju, Y. H. Lee, H. K. Shin, and I. Kim, Appl. Phys. Lett. 71,
}

741 (1997).

${ }^{3}$ Y. G. Ju, J. H. Ser, and Y. H. Lee, IEEE J. Quantum Electron. 33, 589 (1997).

${ }^{4}$ O. Krebs and P. Voisin, Phys. Rev. Lett. 77, 1829 (1996).

${ }^{5}$ O. Krebs, W. Seidel, J. P. André, D. Bertho, C. Jouanin, and P. 
Voisin, Semicond. Sci. Technol. 12, 938 (1997).

${ }^{6}$ O. Krebs, D. Rondi, J. L. Gentner, L. Goldstein, and P. Voisin, Phys. Rev. Lett. 80, 5770 (1998).

${ }^{7}$ R. Magri and S. Ossicini, Phys. Rev. B 63, 165303 (2001).

${ }^{8}$ J. R. Meyer, C. A. Hoffman, F. J. Bartoli, and L. R. Ram-Mohan, Appl. Phys. Lett. 67, 757 (1995).

${ }^{9}$ Q. K. Yang, F. Fuchs, J. Schmitz, and W. Pletschen, Appl. Phys. Lett. 81, 4757 (2002).

${ }^{10}$ L. W. Wang, S. H. Wei, T. Mattila, A. Zunger, I. Vurgaftman, and J. R. Meyer, Phys. Rev. B 60, 5590 (1999).

${ }^{11}$ R. Magri and A. Zunger, Phys. Rev. B 65, 165302 (2002).

${ }^{12}$ F. Szmulowicz, H. J. Haugan, and G. J. Brown, Phys. Rev. B 69, 155321 (2004).

${ }^{13}$ J. T. Olesberg, W. H. Lau, M. E. Flatte, C. Yu, E. Altunkaya, E. M. Shaw, T. C. Hasenberg, and T. F. Boggess, Phys. Rev. B 64, 201301(R) (2001).

${ }^{14}$ K. C. Hall, K. Gündogdu, E. Altunkaya, W. H. Lau, M. E. Flatté, T. F. Boggess, J. J. Zinck, W. B. Barvosa-Carter, and S. L. Skeith, Phys. Rev. B 68, 115311 (2003).

${ }^{15}$ J. N. Schulman and Y. C. Chang, Phys. Rev. B 31, 2056 (1985).

${ }^{16}$ Y. C. Chang and J. N. Schulman, Phys. Rev. B 31, 2069 (1985).

${ }^{17}$ F. Szmulowicz, Phys. Rev. B 54, 11539 (1996).

${ }^{18}$ F. Szmulowicz, Phys. Rev. B 57, 9081 (1998).

${ }^{19}$ E. L. Ivchenko, A. Y. Kaminski, and U. Rössler, Phys. Rev. B 54, 5852 (1996).

${ }^{20}$ V. Mlinar, M. Tadić, B. Partoens, and F. M. Peeters, Phys. Rev. B 71, 205305 (2005).

${ }^{21}$ B. A. Foreman, Phys. Rev. Lett. 81, 425 (1998).

${ }^{22}$ B. H. Hong, S. I. Rybchenko, I. E. Itskevich, S. K. Haywood, R. Intartaglia, V. Tasco, G. Raino, and M. De Giorgi, Phys. Rev. B
79, 165323 (2009).

${ }^{23}$ B. A. Foreman, Phys. Rev. B 56, R12748 (1997).

${ }^{24}$ L. Vervoort, R. Ferreira, and P. Voisin, Semicond. Sci. Technol. 14, 227 (1999).

${ }^{25}$ W. Xu, L. L. Li, H. M. Dong, G. Gumbs, and P. A. Folkes, J. Appl. Phys. 108, 053709 (2010).

${ }^{26}$ L. R. Ram-Mohan, K. H. Yoo, and R. L. Aggarwal, Phys. Rev. B 38, 6151 (1988).

${ }^{27}$ S. L. Chuang, Phys. Rev. B 43, 9649 (1991).

${ }^{28}$ F. M. Peeters and J. T. Devreese, Phys. Rev. B 23, 1936 (1981); Solid State Physics (Elsevier, Amsterdam, The Netherlands, 1984), Vol. 38, p. 81.

${ }^{29}$ W. Xu, Appl. Phys. Lett. 89, 171107 (2006).

${ }^{30}$ X. L. Lei and S. Y. Liu, J. Phys.: Condens. Matter 12, 4655 (2000).

${ }^{31}$ J. R. Waterman, B. V. Shanabrook, R. J. Wagner, M. J. Yang, J. L. Davis, and J. P. Omaggio, Semicond. Sci. Technol. 8, S106 (1993).

${ }^{32}$ H. J. Haugan, F. Szmulowicz, G. J. Brown, and K. Mahalingam, J. Appl. Phys. 96, 2580 (2004).

${ }^{33}$ M. O. Manasreh, F. Szmulowicz, T. Vaughan, K. R. Evans, C. E. Stutz, and D. W. Fischer, Phys. Rev. B 43, 9996 (1991).

${ }^{34}$ T. Unuma, M. Yoshita, T. Noda, H. Sakaki, and H. Akiyama, J. Appl. Phys. 93, 1586 (2003).

${ }^{35}$ J. Li, K. I. Kolokolov, C. Z. Ning, D. C. Larrabee, G. A. Khodaparast, J. Kono, K. Ueda, Y. Nakajima, S. Sasa, and M. Inoue, in Progress in Semiconductors II-Electronics and Optoelectronic Applications, MRS Symposia Proceedings No. 744, edited by B. D. Weaver, M. O. Manasreh, C. C. Jagadish, and S. Zollner (Materials Research Society, Pittsburgh, PA, 2003), pp. M9.2.1M9.2.12. 